\title{
CORRIGENDUM
}

\section{Genetic contributions to self-reported tiredness}

V Deary, SP Hagenaars, SE Harris, WD Hill, G Davies, DCM Liewald, International Consortium for Blood Pressure GWAS, CHARGE consortium Aging and Longevity Group, CHARGE consortium Inflammation Group, AM Mclntosh, CR Gale and IJ Deary

Molecular Psychiatry (2018) 23, 789-790; doi:10.1038/mp.2017.70; published online 21 March 2017

Correction to: Molecular Psychiatry (2017) advance online publication 14 February 2017; doi:10.1038/mp.2017.5

Following publication of this paper, the authors noticed a mistake in Tables 3 and 4 and Figure 3 . The genetic correlation between tiredness and C-reactive protein was displayed as 0.0165 , but the correct genetic correlation is 0.1650 . The thresholds for major depressive disorder and neuroticism are incorrect in Table 4. The threshold for major depressive disorder is 0.1 , and for neuroticism the threshold is 1 . The corrected tables and figure appear below.

\begin{abstract}
(i) This work is licensed under a Creative Commons Attribution 4.0 International License. The images or other third party material in this article are included in the article's Creative Commons license, unless indicated otherwise in the credit line; if the material is not included under the Creative Commons license, users will need to obtain permission from the license holder to reproduce the material. To view a copy of this license, visit http://creativecommons.org/licenses/ by/4.0/
\end{abstract}

(c) The Author(s) 2018

Table 3. Genetic correlations between tiredness documented in the UK Biobank data set and the health-related variables collected from GWAS consortia

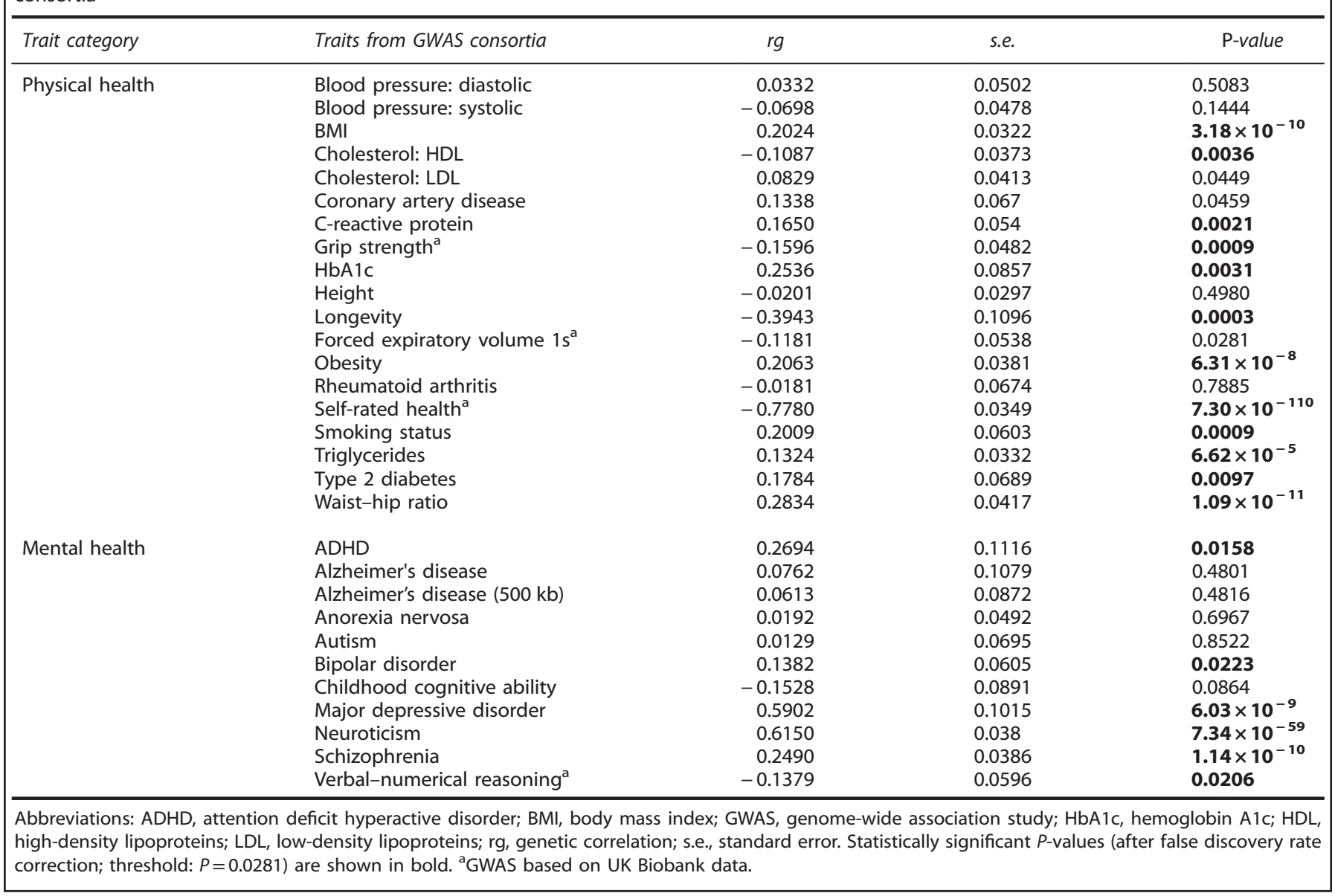


Table 4. Associations between polygenic profile scores of health-related traits created from GWAS consortia summary data, and the UK Biobank tiredness phenotype controlling for age, sex, assessment centre, genotyping batch and array, and 10 principal components for population structure

\begin{tabular}{|c|c|c|c|c|}
\hline Trait category & Trait & Threshold & $\beta$ & P-value \\
\hline \multirow[t]{17}{*}{ Physical health } & Blood pressure: diastolic & 0.1 & -0.0028 & 0.3619 \\
\hline & Blood pressure: systolic & 0.1 & -0.0025 & 0.4077 \\
\hline & $\mathrm{BMI}$ & 1 & 0.0280 & $4.90 \times 10^{-20 a}$ \\
\hline & Cholesterol: HDL & 0.5 & -0.0163 & $8.49 \times 10^{-8 a}$ \\
\hline & Cholesterol: LDL & 0.5 & 0.0081 & $0.0077^{\mathrm{a}}$ \\
\hline & Coronary artery disease & 0.5 & 0.0084 & 0.0061 \\
\hline & C-reactive protein & 1 & 0.0130 & $2.10 \times 10^{-5 a}$ \\
\hline & Forced expiratory volume $1 \mathrm{~s}$ & 0.01 & -0.0059 & 0.0529 \\
\hline & Longevity & 0.05 & -0.0067 & 0.0297 \\
\hline & $\mathrm{HbA} 1 \mathrm{c}$ & 1 & 0.0090 & $0.0033^{a}$ \\
\hline & Height & 1 & -0.0077 & 0.0154 \\
\hline & Obesity & 1 & 0.0236 & $1.20 \times 10^{-14 a}$ \\
\hline & Rheumatoid arthritis & 0.1 & -0.0016 & 0.5926 \\
\hline & Smoking status & 0.5 & 0.0086 & 0.0071 \\
\hline & Triglycerides & 0.5 & 0.0209 & $1.06 \times 10^{-11 a}$ \\
\hline & Type 2 diabetes & 1 & 0.0120 & $0.0002^{a, b}$ \\
\hline & Waist-hip ratio & 1 & 0.0258 & $7.85 \times 10^{-17 a}$ \\
\hline \multirow[t]{9}{*}{ Mental health } & ADHD & 1 & 0.0042 & 0.1647 \\
\hline & Alzheimer's disease & 0.05 & -0.0052 & 0.0889 \\
\hline & Anorexia nervosa & 0.5 & 0.0048 & 0.1169 \\
\hline & Autism & 1 & -0.0018 & 0.5593 \\
\hline & Bipolar disorder & 0.01 & 0.0081 & $0.0076^{c}$ \\
\hline & Childhood cognitive ability & 0.1 & -0.0112 & $0.0002^{\mathrm{a}}$ \\
\hline & Major depressive disorder & 0.1 & 0.0185 & $2.25 \times 10^{-9 c}$ \\
\hline & Neuroticism & 1 & 0.0183 & $2.00 \times 10^{-9 c}$ \\
\hline & Schizophrenia & 1 & 0.0283 & $2.31 \times 10^{-19 a, c}$ \\
\hline
\end{tabular}

Abbreviations: ADHD, attention deficit hyperactive disorder; BMI, body mass index; GWAS, genome-wide association study; HbA1c, hemoglobin A1c; HDL, high-density lipoproteins; LDL, low-density lipoproteins. False discovery rate-corrected statistically significant values $(P=0.0255)$ are shown in bold. The associations between the polygenic profile scores with the largest effect size (threshold) and tiredness are presented. Threshold is the $P$-value threshold with the largest effect size. ${ }^{a}$ Results remain significant after controlling for neuroticism scores. ${ }^{b}$ Results remain significant after excluding individuals with type 2 diabetes $(\beta=0.0105, P=0.00076)$. ${ }^{c}$ Results remain significant after controlling for self-rated health.

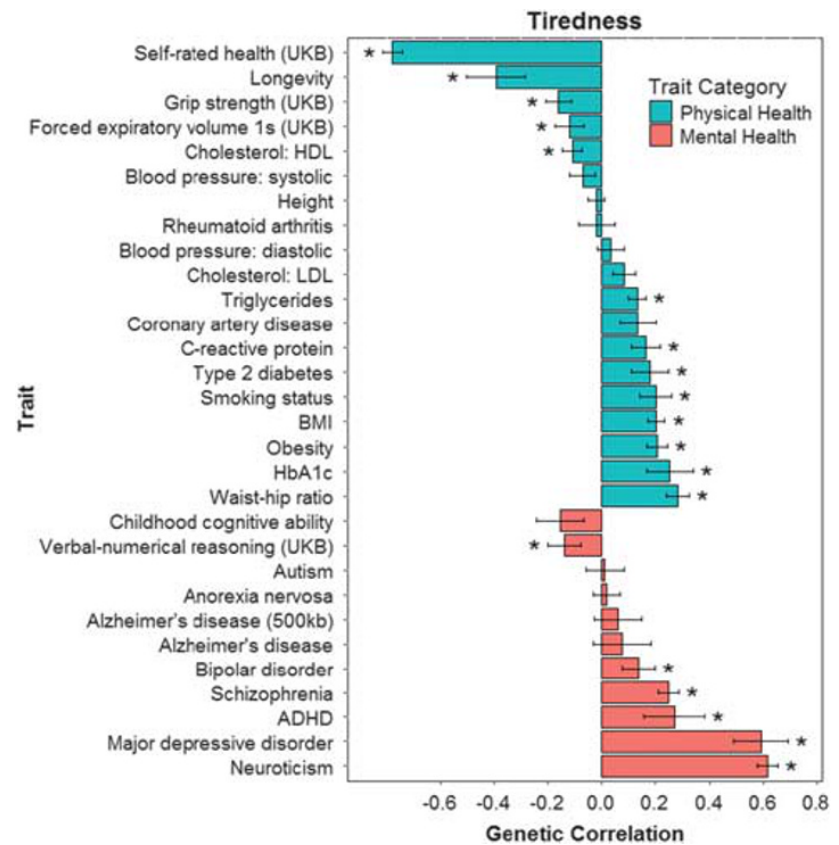

Figure 3. Barplot of genetic correlations (s.e.) calculated using linkage disequilibrium score regression between tiredness in UK Biobank, and mental and physical health measures from GWAS consortia. ${ }^{*} P<0.0281$. ADHD, attention deficit hyperactive disorder; BMI, body mass index; GWAS, genome-wide association study; $\mathrm{HbA1c}$, hemoglobin A1c; HDL, high-density lipoproteins; LDL, lowdensity lipoproteins. 